\title{
PROMOVENDO A APRENDIZAGEM COLABORATIVA COM MAPAS CONCEITUAIS NAS AULAS DE GEOGRAFIA
}

Prof. Ms. Leandro Fabrício Campelo

campelo@ifsp.edu.br

Doutorando do PPGE da Universidade de São Paulo- USP, Docente do Instituto Federal de São Paulo (Campus Cubatão) 
RESUMO: Mapas conceituais criam oportunidades para os alunos mostrarem relações significativas entre os conceitos estudados na Geografia. Trata-se de uma estratégia adequada a mudança do paradigma da avaliação tradicional para os processos de aprendizagem qualitativa e formativa. Sua lógica baseia-se na teoria da aprendizagem cognitiva de David Ausubel. É uma técnica desenvolvida em meados dos anos setenta por Joseph Novak e seus colegas na Universidade de Cornell. Neste artigo, apresentamos os resultados de pesquisas realizadas sobre o ensino e aprendizagem de conceitos geográficos com 38 alunos do Ensino Médio. Inicialmente, os alunos criaram os mapas conceituais no papel, posteriormente, eles foram subdivididos em pares e com o uso do CmapTools, de forma colaborativa, criou-se a oportunidade de gerar um novo mapa conceitual, analisado mediante o uso do peer review. Os resultados mostraram mapas de qualidade conceitual significativamente melhores do que quando construídos individualmente, indicando uma compreensão conceitual mais significativa e profunda.

PALAVRAS-CHAVE: ensino de geografia, mapas conceituais, aprendizagem colaborativa.

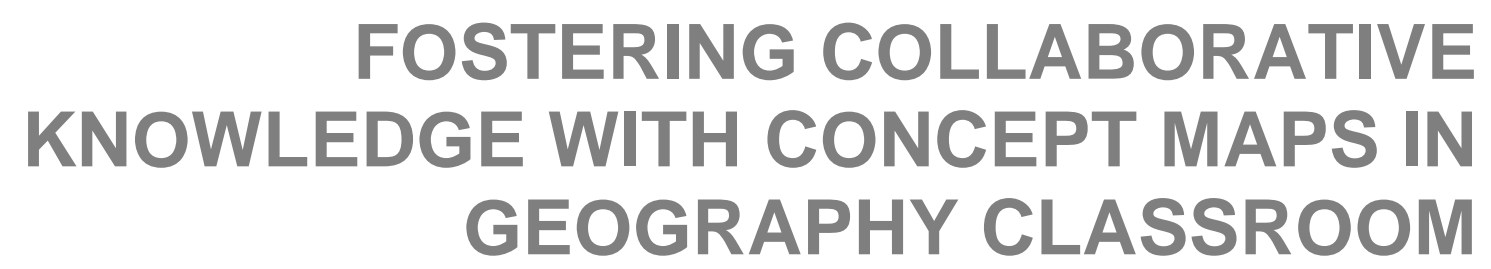

ABSTRACT: Concept maps create opportunities for students to show meaningful relationships between concepts taught in Geography. It is a strategy appropriate to the paradigm shift from traditional assessment to qualitative and formative learning processes. Its rationale is based on David Ausubel cognitive learning theory. It is a technique developed in the mid-seventies by Joseph Novak and his colleagues at Cornell University. In this paper, we present the results of research carried out on the teaching and learning of geographic concepts with 38 students of a High School. Initially the students individually created the concept maps on paper before presenting the key concepts to the teacher. Subsequently, they were subdivided into pairs. The CmapTools application, in a collaborative way, created the opportunity to create a new concept map that was analyzed and peer-review. Results showed significantly improved conceptual quality maps than when constructed individually, thus indicating more meaningful and profound conceptual understanding.

KEYWORDS: geography, concept maps, collaborative learning. 


\section{Introdução}

A aprendizagem envolve muito mais do que a capacidade de recordar informações, fatos e nomes (CORREIA, CABRAL e AGUIAR, 2016). Os mapas conceituais (MC) são amplamente utilizados em todo o mundo por pessoas de todas as idades para expressar a sua compreensão sobre diversos domínios do conhecimento (CAÑAS, NOVAK e REISKA, 2015). Os mapas conceituais consistem em rótulos conceituais que identificam ideias específicas (conceitos) e as ligações entre eles, o que explica como os conceitos estão relacionados para criar significado. Um par de conceitos e suas respectivas ligações fazem uma única proposição (conceito inicial - termo de ligação $\rightarrow$ conceito final), assim, um mapa conceitual é feito de qualquer número de proposições para dar uma definição pessoal de qualquer ideia ou fenômeno particular (NOVAK, 2010).

Os MC são possivelmente uma das melhores ferramentas para aumentar a visibilidade das ideias complexas para facilitar a discussão entre professor e aluno sobre um determinado tema estudado. A estratégia é mais rigorosa do que o mapa mental, tratase de uma poderosa ferramenta de ensino, uma vez que facilita a representação dos modelos mentais do aluno. Alguns trabalhos sobre mapas conceituais consideram a importância do mapeamento conceitual colaborativo (KINCHIN, DELEIJ e HAY, 2005; BOXTEL, LINDEN, ROELOFS e ERKENS, 2010; TORRES e MARRIOT, 2010; MOON, HANSBERGER e TATE, 2011), com particular referência à promoção do diálogo entre professores e alunos (KINCHIN, 2003; HAY, 2008).

Essa maneira de representar o conhecimento pode ser enriquecida com um software, o CmapTools ${ }^{1}$, que facilita a criação dos MC e que também amplia o leque de possibilidades, como por exemplo, de utilização de som, vídeo, imagem etc. O CmapTools é o principal programa para a criação de mapas conceituais e foi desenvolvido no Florida Institute for Human \& Machine Cognition (IHMC), localizado nos Estados Unidos. Reúne os pontos fortes dos mapas conceituais com o poder dos computadores e da internet. Esse software permite ao usuário criar MC e refiná-los muito facilmente, é projetado para pessoas de qualquer idade que podem compartilhar seus mapas conceituais com mapeadores e leigos em todo o mundo.

\footnotetext{
${ }^{1}$ Disponível para download em <https://www.ihmc.us/cmaptools/>.
} 
O conhecimento científico sobre os processos de construção colaborativa do conhecimento nos ajuda a apoiar os alunos de forma mais eficaz em situações de aprendizagem colaborativa (FISCHER, BRUHN, GRASEL e MANDL, 2002). O desenvolvimento de tal conhecimento construído e reconstruído pode ser representado graficamente usando os MC. A ação do mapeamento conceitual também é pensada para ajudar o processo, revelando às conexões dos alunos que não tinham sido reconhecidas anteriormente e atuando como um foco para a comunicação entre o aluno e o professor (KINCHIN e HAY, 2000).

\section{Construções do Conhecimento Colaborativo}

"Construção do conhecimento colaborativo" e "Construção de conhecimento" são exemplos de termos comumente usados na pesquisa para descrever o processo cognitivo relevante para a aprendizagem colaborativa. Com base na teoria existente, temos quatro processos de construção de conhecimento colaborativo: a) Externalização, b) Elicitação, c) Consenso orientado para o conflito, d) Consenso orientado para a integração (FISCHER, BRUHN, GRASEL e MANDL, 2002). Estes serão descritos abaixo:

a) Externalização: algumas pesquisas mostram que para resolver um problema trabalhando colaborativamente é fundamental expor o conhecimento dos alunos envolvidos. Uma condição necessária para uma construção colaborativa do conhecimento ocorre quando os alunos discutem e apresentam o seu conhecimento prévio sobre o tema a ser resolvido, desta forma, as diferentes opiniões serão expostas (BROWN, COLLINS e DUGUID, 1989).

b) Elicitação: um aspecto muito importante da construção do conhecimento colaborativo é fazer com que os alunos expressem os seus conhecimentos relacionados à tarefa. Assim, os outros alunos podem aprender com o conhecimento do parceiro. Isso é referido como "usar o parceiro de aprendizagem como um recurso" (DILLENBOURG, BAKER, BLAYE e O’MALLEY, 1995). Durante a discussão para resolver uma tarefa específica, os alunos usam o que outros alunos expressam para externalizar o que sabem sobre o tópico.

c) Consenso orientado para o conflito: na discussão orientada para o conflito, diferentes ideias e entendimentos sobre um tema estimulam o debate e através deste conflito os alunos podem chegar a uma resolução da tarefa proposta. As diferentes 
interpretações feitas pelos parceiros de aprendizagem estimulam processos que podem levar a uma modificação das estruturas do conhecimento (DILLENBOURG, 1999).

d) Consenso orientado para a integração: a discussão orientada para a integração é outra maneira de alcançar um consenso, integrando as diferentes perspectivas individuais em uma interpretação ou solução comum da tarefa dada. Esta forma de construção de consenso pode ser importante sob certas condições. No entanto, uma tentativa de incorporar todas as sugestões em apenas uma perspectiva comum pode levar a uma resposta superficial. Alguns estudantes falham em declarar sua opinião para evitar conflitos. Poder-se-ia falar de uma tendência dos aprendentes para chegar a um consenso ilusório (FISCHER, BRUHN, GRASEL e MANDL, 2002).

\subsection{Interação colaborativa}

Outro ponto importante na construção do conhecimento colaborativo é o tipo de interação que ocorre na sala de aula. Três tipos diferentes de interação colaborativa podem ser identificados durante o processo de aprendizagem: a) A colaboração vertical ocorrida entre professor e aluno prevalece nas salas de aula tradicionais, onde a tarefa do segundo é receber o conhecimento transmitido; b) Uma colaboração horizontal é observada quando os alunos interagem com os seus pares; c) Uma condição intermediária pode ser criada quando o professor e os alunos interagem em um ambiente de aprendizagem construtivista, a colaboração diagonal pode ser considerada uma situação híbrida de colaboração vertical e horizontal (CORREIA, CICUTO, e AGUIAR, 2014). Neste estudo foi utilizada uma colaboração diagonal.

\subsection{Outros estudos}

Ao longo do século passado, os pesquisadores da área da Psicologia, como Piaget e Vygotsky, sublinharam a importância da interação entre os estados sociais, afetivos e cognitivos no desenvolvimento e na aprendizagem e, portanto, forneceram uma fundamentação teórica para o uso de grupos em configurações de instrução. Essas ideias têm promovido a visão de que o pensamento das crianças é uma função do conhecimento prévio e da capacidade do indivíduo para aprender com a ajuda de adultos ou colegas e, como resultado, levou a uma ênfase nos benefícios da formação em pares, aprendizagem colaborativa e cooperativa para desenvolvimento cognitivo. As atividades colaborativas são importantes para permitir o intercâmbio social durante o processo de aprendizagem e devem ser usadas com mais frequência no ambiente escolar. 
Novos desafios são apresentados ao sistema educacional e uma revisão dos procedimentos metodológicos é imprescindível para preparar os cidadãos capazes de lidar com questões contemporâneas complexas.

Num outro estudo, elaborado por Correia e Malachias (2010), foi criada uma atmosfera verdadeiramente colaborativa e condição favorável para empoderar os alunos, estimulando-os a agir como cidadãos autônomos. A avaliação de pares foi utilizada numa situação em que os alunos avaliaram os mapas conceituais feitos por seus pares. As características qualitativas sugerem que a colaboração e a avaliação por pares podem apoiar o desenvolvimento de melhores mapas conceituais em comparação com a produção individual.

Em um grande estudo desenvolvido no Reino Unido, os pesquisadores mostram a importância do trabalho colaborativo, mas argumentam que para ser produtivo, o professor deve estar bem preparado para desenvolver essa estratégia. A sala de aula do futuro precisa de mudanças e o trabalho colaborativo pode colaborar para resolver os problemas que surgirão no século XXI (BLATCHFORD, P.; KUTNICK, P.; BAINES, E. e GALTO, 2003).

\section{Aprendizagem significativa e mapeamento conceiltual}

O conceito primordial da teoria de Ausubel é o de aprendizagem significativa, que ocorre quando uma nova informação que apresenta uma estrutura lógica interage com conceitos relevantes e inclusivos, claros e disponíveis na estrutura cognitiva do aprendiz, sendo por ele assimilado, contribuindo para sua diferenciação, elaboração e estabilidade. Essa interação constitui uma experiência consciente, claramente articulada e precisamente diferenciada, que emerge quando sinais, símbolos, conceitos e proposições potencialmente significativos são relacionados à estrutura cognitiva e nela incorporados (AUSUBEL, 2000).

Os conceitos que interagem com novos conhecimentos constituem a base para a atribuição de novos significados. Vão se modificando em função dessa interação, adquirindo novos significados e se diferenciando progressivamente. Esse processo é chamado de diferenciação progressiva. Quando um aluno aprende sobre as estações do ano nas aulas de Geografia, considera-se que, a princípio, quanto mais próxima da Terra estiver do Sol, a temperatura será mais alta. Quando se tem acesso a novas informações, como o movimento de translação e a inclinação do eixo de rotação da Terra, os alunos 
diferenciam progressivamente o entendimento anterior para outro mais próximo da informação científica. O movimento de translação, associado à inclinação do eixo de rotação da Terra, leva a ocorrência das estações do ano.

Outro processo que ocorre no curso da aprendizagem significativa é o estabelecimento de relações entre ideias, conceitos, proposições já estabelecidas na estrutura cognitiva, por exemplo, relações entre subsunçores. Elementos existentes na estrutura cognitiva com determinado grau de clareza, estabilidade e diferenciação são percebidos como relacionados, adquirem novos significados e levam a uma reorganização da estrutura cognitiva. Essa recombinação e/ou reorganização cognitiva estabelece uma relação significativa, conhecida como reconciliação integrativa. Toda aprendizagem que resultar em reconciliação integrativa resultará também em diferenciação progressiva adicional de conceitos e proposições. A reconciliação integrativa é uma forma de diferenciação progressiva da estrutura cognitiva. É um processo cujo resultado é o explícito delineamento de diferenças e de similaridades entre ideias relacionadas (MOREIRA e MASINI, 2006).

A aprendizagem significativa acontece quando o aluno escolhe relacionar novas informações com o conhecimento que já possui. A sua qualidade também depende da riqueza conceitual do novo material a ser aprendido e da quantidade e qualidade da organização do conhecimento relevante detido pelo aluno. Para Ausubel (2000), o aprendizado mecânico ocorre quando o aprendiz memoriza novas informações sem relacioná-lo com o conhecimento prévio. A aprendizagem significativa tem três requisitos: a) conhecimento prévio relevante - o aluno deve conhecer algumas informações relacionadas às novas informações a serem aprendidas; b) material significativo - o conhecimento a ser aprendido deve ser relevante para outros conhecimentos e deve conter conceitos e proposições significativas; c) o aluno deve escolher aprender de forma significativa - o aluno deve conscientemente e deliberadamente escolher relacionar novos conhecimentos com o conhecimento relevante que ele já conhece (NOVAK, 2010).

\subsection{A criação dos mapas conceituais}

Durante o desenvolvimento de um projeto de ensino com áudio e tutorial nos anos de 1970, o professor Joseph Novak e seus colaboradores, tendo a necessidade de interpretar o conteúdo das gravações e descobrir os padrões de mudança na compreensão 
conceitual dos alunos sobre ciências, começaram a representar as estruturas cognitivas por meio de mapas hierarquizados de conceitos e de proposições (NOVAK e MUSONDA, 1991). Dessa forma, os primeiros mapas conceituais foram construídos a partir de informações das entrevistas com alunos e só depois foram utilizados nas salas de aula para serem produzidos pelos próprios alunos, de modo a entender suas estruturas cognitivas.

De acordo com Novak e Cañas (2010, p.10), “[...] mapas conceituais são ferramentas gráficas para a organização e representação do conhecimento". Os mapas conceituais devem incluir conceitos que ficam dentro de círculos ou quadrados, as relações entre estes conceitos são indicadas por linhas que os interligam. As palavras sobre as linhas são frases de ligação que especificam os relacionamentos entres os conceitos.

Segundo Novak (2010), uma das funções mais importantes que os mapas conceituais podem desempenhar é ajudar um grupo a capturar, e chegar a um consenso sobre, o conhecimento coletivo sobre alguma questão ou conjunto de questões de interesse para o grupo. Grandes projetos colaborativos já foram desenvolvidos com a NASA e o Serviço Meteorológico Nacional, onde os mapas conceituais suportados pelo programa CmapTools geraram excelentes resultados do trabalho colaborativo.

Os que usam MC em sala de aula percebem que alguns alunos têm dificuldade em elaborar e usar mapas conceituais, pelo menos no início, isso é explicado por anos de aprendizado mecânico (decoreba), que ainda prevalece em muitas escolas. Dessa forma, não será fácil para um aluno trocar rapidamente anos de aprendizado mecânico por uma aprendizagem significativa, mas isso é importante, uma vez que o aprendizado mecânico contribui muito pouco para a estrutura do conhecimento, por isso não é a base para o pensamento criativo.

\section{Avaliando um mapa conceitual}

Na Tabela 1, apresentamos uma proposta elaborada por Cañas, Novak e Reiska (2015) para classificar a qualidade de um mapa conceitual, pensando na qualidade estrutural e do conteúdo. Quando nos referimos a estrutura de um mapa conceitual pensamos em suas ligações cruzadas, conceitos, proposições etc. Já o conteúdo é relacionado ao que se está sendo desenvolvido no mapa conceitual, é importante destacar 
que todo mapa conceitual deve ter uma pergunta focal, o que limita o tema e o conteúdo a ser apresentado.

Um mapa conceitual que tenha uma "boa" qualidade estrutural, mas a qualidade de conteúdo é "fraca", ou vice-versa, são considerados como um mapa conceitual "pobre" de Nível 1. Enquanto um mapa conceitual com "boa" qualidade estrutural e "boa" qualidade de conteúdo é classificado como um MC de Nível 2.

\section{Tabela 1: Como avaliar a qualidade de um mapa conceitual}

\begin{tabular}{llccc}
\hline Nível de Qualidade & $\begin{array}{c}\text { Qualidade da } \\
\text { Estrutura }\end{array}$ & $\begin{array}{c}\text { Qualidade do } \\
\text { Conteúdo }\end{array}$ & $\begin{array}{c}\text { Qualidade do Mapa } \\
\text { Conceitual }\end{array}$ \\
\hline 1 & Pobre & $\sqrt{ }$ & - & - \\
& & - & $\sqrt{ }$ & - \\
2 & Bom & $\sqrt{ }$ & $\sqrt{ }$ & $\sqrt{ }$ \\
3 & Excelente & $\sqrt{ }$ & $\sqrt{ }$ & Fonte: Cañas, Novak e Reiska (2015, p.11)
\end{tabular}

Com relação ao Nível 3, na comunidade de mapeadores (Cmappers), ainda não há um consenso sobre este nível, mas já existem algumas pesquisas (CAÑAS, REISKA e NOVAK, 2016; SCHWENDIMANN, 2016; NOVAK e CAÑAS, 2010) que procuram aprofundar o debate.

Avaliar um MC não é uma tarefa fácil. Numa sala de aula com 30 alunos, por exemplo, o professor terá que analisar cada mapa e devolver um feedback para cada aluno. Mas o resultado é muito positivo, pois podemos entender o que o aluno sabe ou não sobre determinado assunto que está sendo estudado, assim, o professor poderá através da análise dos MC rever pontos importantes da disciplina.

\section{O Ensino de Geografia}

Diversos especialistas sobre o ensino de Geografia (CASTELLAR, 2014; CASTELLAR e VILHENA, 2010; CAVALCANTI, 2013; MAIA, 2014; KIMURA, 2008; VENTURI, 2011; REGO, CASTROGIOVANNI, KAECHER， 2011; CASTROGIOVANNI et al. 2014) recomendam a revisão das concepções teóricometodológicas da ciência geográfica com objetivo de propor situações de aprendizagem que superem o ensino tradicional pautado na memorização de fatos e do senso comum que ainda perdura no ensino de Geografia.

Tanto o aluno quanto o professor de Geografia precisam entender o mundo globalizado e complexo que vivemos, que acontecimentos locais estão emaranhados em 
acontecimentos globais e muitas vezes não conseguimos separar um fato do outro. A Geografia precisa mostrar força neste momento, como apontou Souza Santos (2007). Vivemos num momento de perguntas fortes, porém de respostas fracas. Hammes, Forster e Chaigar (2014) consideram que existem, de fato, perguntas fortes para a Geografia, como as que questionam a escola e seu papel num contexto difícil de consumismo exacerbado e complexidade das relações sociais.

Para repensar o ensino de Geografia em um mundo em constantes modificações, precisamos de metodologias de ensino que favoreçam o ensino e a aprendizagem, como é o caso dos mapas conceituais, que utilizado nos computadores por meio do software CmapTools, associado a um trabalho colaborativo, com uma avaliação inovadora nas salas de aula (peer review) podem favorecer uma aprendizagem significativa e tornar os alunos protagonistas na sala de aula.

\section{Método}

\subsection{Participantes}

Participaram dessa pesquisa 38 alunos do $1^{\circ}$ ano do Ensino Médio do Instituto Federal de São Paulo, Câmpus Suzano. O estudo foi realizado durante dois meses nas aulas de Geografia e os participantes foram divididos em duplas numa etapa da pesquisa. Essas duplas foram criadas aleatoriamente, de modo que nunca haviam trabalhado juntas anteriormente. Todos os alunos receberam informações de como trabalhar de forma colaborativa.

\subsection{Procedimentos}

A pesquisa tem uma abordagem qualitativa, de natureza configurada como pesquisa-ação. A pesquisa-ação está associada a várias formas de ação coletiva que podem ser orientadas para metas de transformação ou resolução de problemas. Diversas áreas do conhecimento usam essa abordagem, como a Educação, o Serviço Social e a Comunicação.

A pesquisa-ação visa ao conhecimento situacional de um problema em seu contexto e busca os meios ou procedimentos disponíveis para realizá-lo. Pretende compreender uma situação prática e a proposição de solução alternativa ao problema que a investigação coloca. Isso resulta em ação complexa, que merece uma investigação planejada e conduzida. Na pesquisa-ação, o pesquisador tem um papel importante e ativo 
para resolver os problemas que surgem, como monitorar e avaliar todas as ações desencadeadas pelos desafios encontrados.

$\mathrm{Na}$ pesquisa, a primeira etapa foi fazer com que os alunos aprendessem sobre a técnica de mapeamento conceitual e como trabalhar de forma colaborativa para resolver uma determinada tarefa. Em seguida, os alunos estudaram temas da Geologia com o apoio do livro didático, vídeos, aulas expositivas etc. Então, individualmente, os alunos criaram o mapa conceitual 1 (MC1) sobre um tópico da Geologia usando papel e caneta. Todos os MC1 foram analisados em detalhes pelo professor e os alunos receberam um feedback sobre a técnica de mapeamento conceitual. O professor também fez comentários com toda a turma explicando os principais erros relacionados ao conteúdo estudado e as técnicas de mapeamento conceitual.

Na segunda etapa, os alunos foram divididos em pares e usando o feedback do $\mathrm{MC} 1$, trabalharam de forma colaborativa e criaram o mapa conceitual 2 (MC2), o que seria uma versão melhorada sobre o $\mathrm{MC1}$, mas agora usaram o programa CmapTools para elaborar o MC2. As interações de duas duplas foram gravadas e analisadas. Depois de recolher o MC2 criado pelos alunos, numa terceira etapa, eles participaram de um peer review duplo cego, uma revisão por pares onde cada dupla analisou dois MC2 de outras duplas. Tanto quem analisou quanto quem foi analisado tiveram seus nomes mantidos em segredo. Por fim, os alunos receberam as sugestões elaboradas pelos seus pares e poderiam aceita-las ou não.

\section{Resultados}

O primeiro mapa conceitual elaborado pelos alunos mostra alguns erros na técnica de mapeamento conceitual, isso é comum porque é a primeira vez que os alunos usaram essa metodologia. Para desenvolver bons mapas conceituais é necessário começar com um conteúdo que os alunos dominam, e isso foi feito. Os alunos fizeram o MC1 depois de estudar o conteúdo sobre Geologia. Uma questão focal também foi usada para selecionar um domínio de conhecimento limitado para os primeiros mapas conceituais. Em seguida, analisaremos um dos pares que tiveram seu trabalho gravado durante a elaboração.

A Figura 1 representa um MC1 desenvolvido pelo estudante Luiz. A pergunta focal era: Qual a relação entre as placas tectônicas e os terremotos" O conteúdo do MC1 ainda é "pobre", o aluno não conseguiu responder adequadamente à pergunta focal. No 
entanto, sua estrutura é "boa", o conceito mais geral está no topo do MC e os conceitos mais específicos no final, além disso o aluno usou adequadamente as setas e os termos de conexão.

\section{Figura 1: MC 1 criado por Luiz}

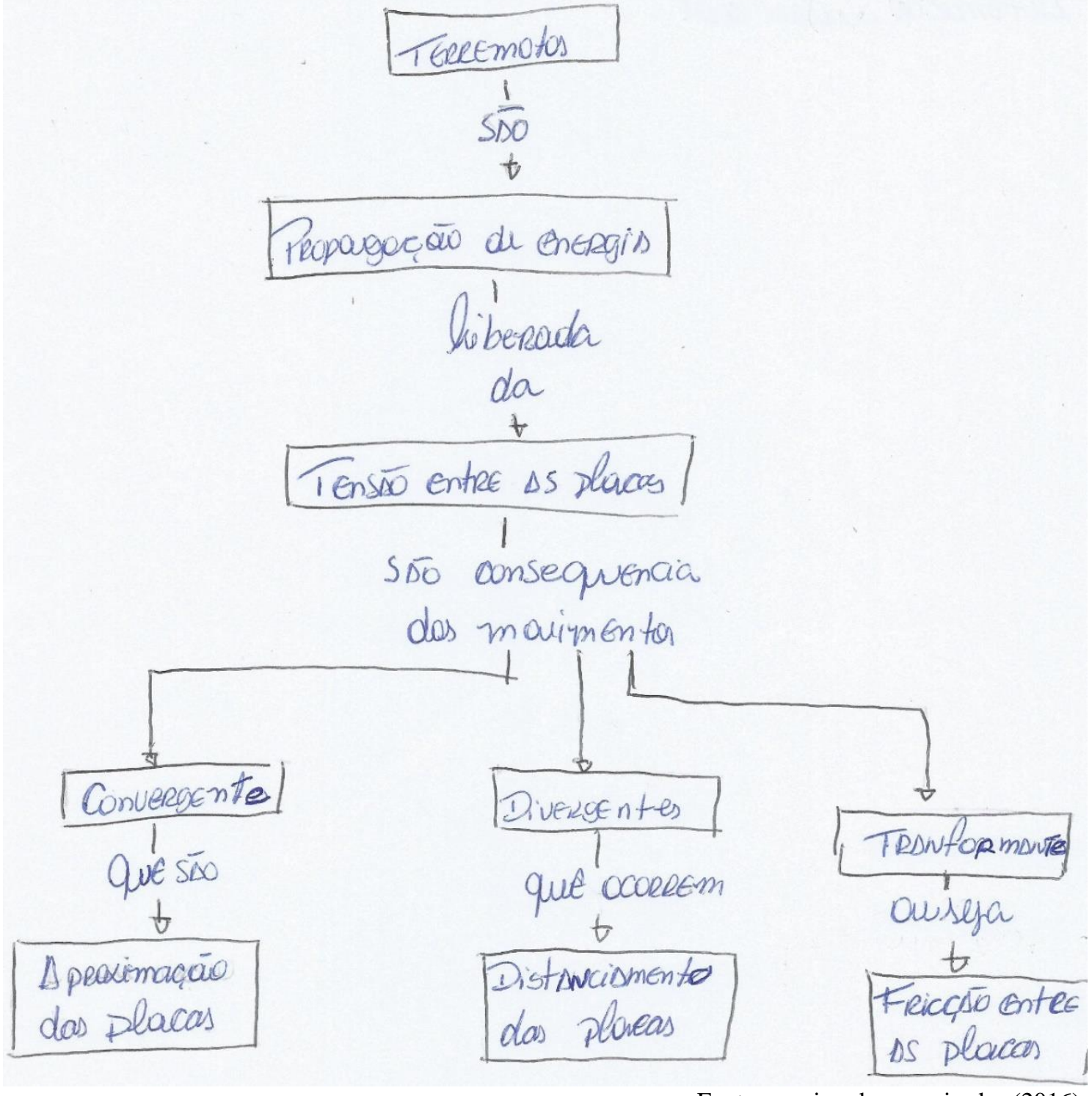

Fonte: arquivo do pesquisador (2016).

Mas o aluno ainda não conseguiu desenvolver ligações cruzadas entre os conceitos em seu primeiro MC1. É necessário mais treinamento na técnica de mapeamento conceitual para aprofundar o domínio sobre o assunto. Assim, podemos classificar MC1 como "pobre". 
A Figura 2 representa outro MC1 do aluno José. Tanto a estrutura quanto o conteúdo são "pobres". O aluno não respondeu adequadamente à pergunta focal (a mesma da Figura 1), usando poucos conceitos estudados.

Em relação à estrutura, o aluno usou as setas de forma errada, além do que o aluno não conseguiu fazer ligações cruzadas entre os conceitos. No entanto, é necessário lembrar que os alunos são iniciantes na técnica de mapeamento conceitual, por isso, cabe ao professor fornecer feedback individual e coletivo para resolver problemas relacionados ao conteúdo (Geologia) e à estrutura do mapa conceitual.

\section{Figura 2: MC 1 criado por José}

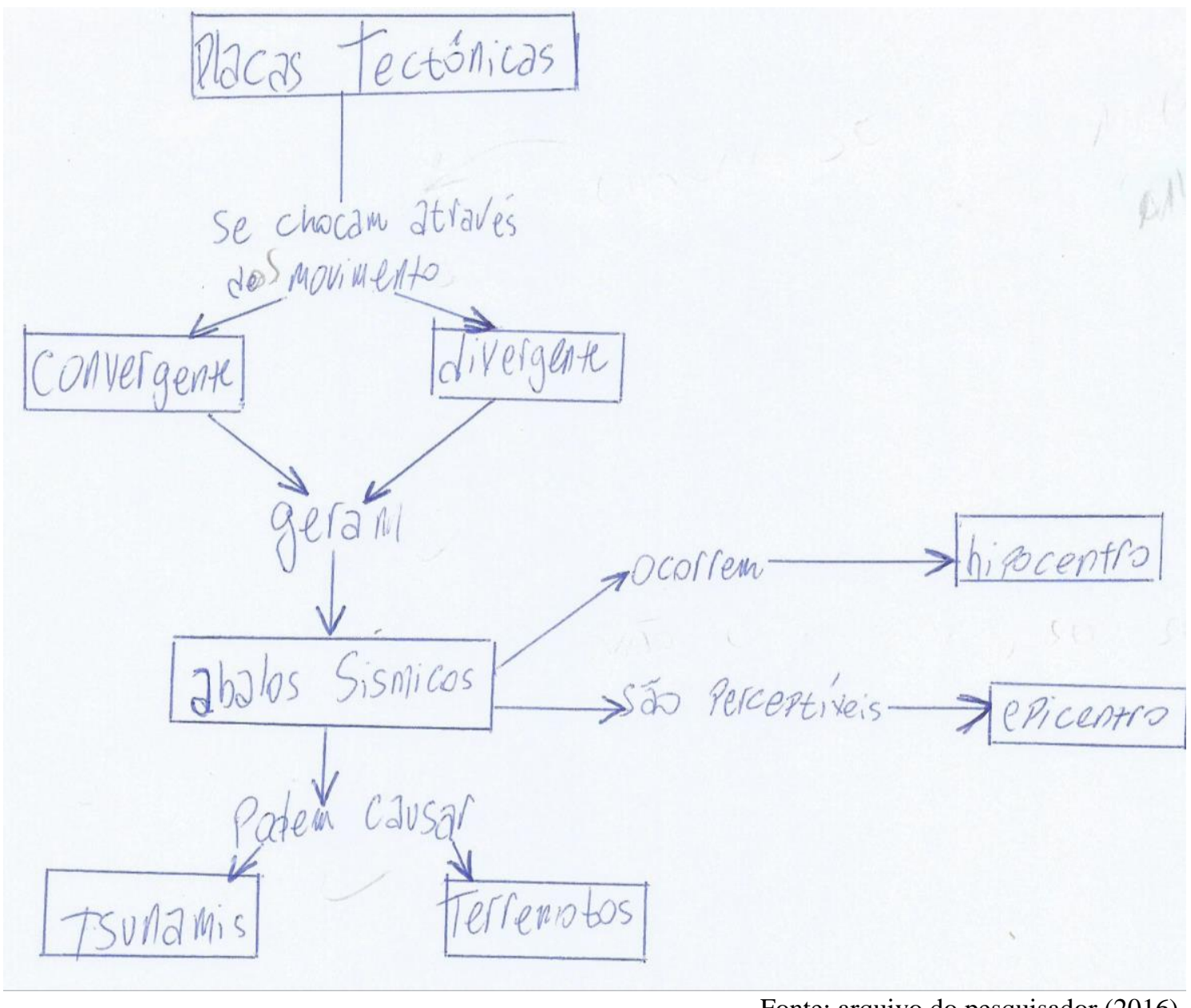

Embora o treinamento na técnica de mapeamento conceitual seja fundamental, é importante destacar, que alguns alunos podem desenvolver essa habilidade melhor, enquanto outros estudantes produzem textos com maior facilidade. Em outro estudo, mostramos que esse tempo é fundamental para melhorar a aprendizagem da técnica de 
mapeamento conceitual e também para alcançar uma aprendizagem significativa sobre um certo conteúdo (CAMPELO e PICONEZ, 2016).

Na Figura 3, observamos o trabalho colaborativo feito pelos alunos Luiz e José usando o programa CmapTools. Ao analisar a gravação da dupla, percebe-se que os alunos desenvolveram uma rica discussão para chegar ao resultado final. Houve uma "Elicitação" e uma "Externalização" favorecendo um "Consenso orientado para conflitos", os alunos discutiram o assunto para chegar a uma versão final do mapa conceitual.

Outro ponto importante foi a "colaboração diagonal", quando professor e alunos interagem em um ambiente de aprendizagem construtivista, o ambiente de ensino favoreceu a aprendizagem significativa.

\section{Figura 3: MC 2 criado por Luiz e José}

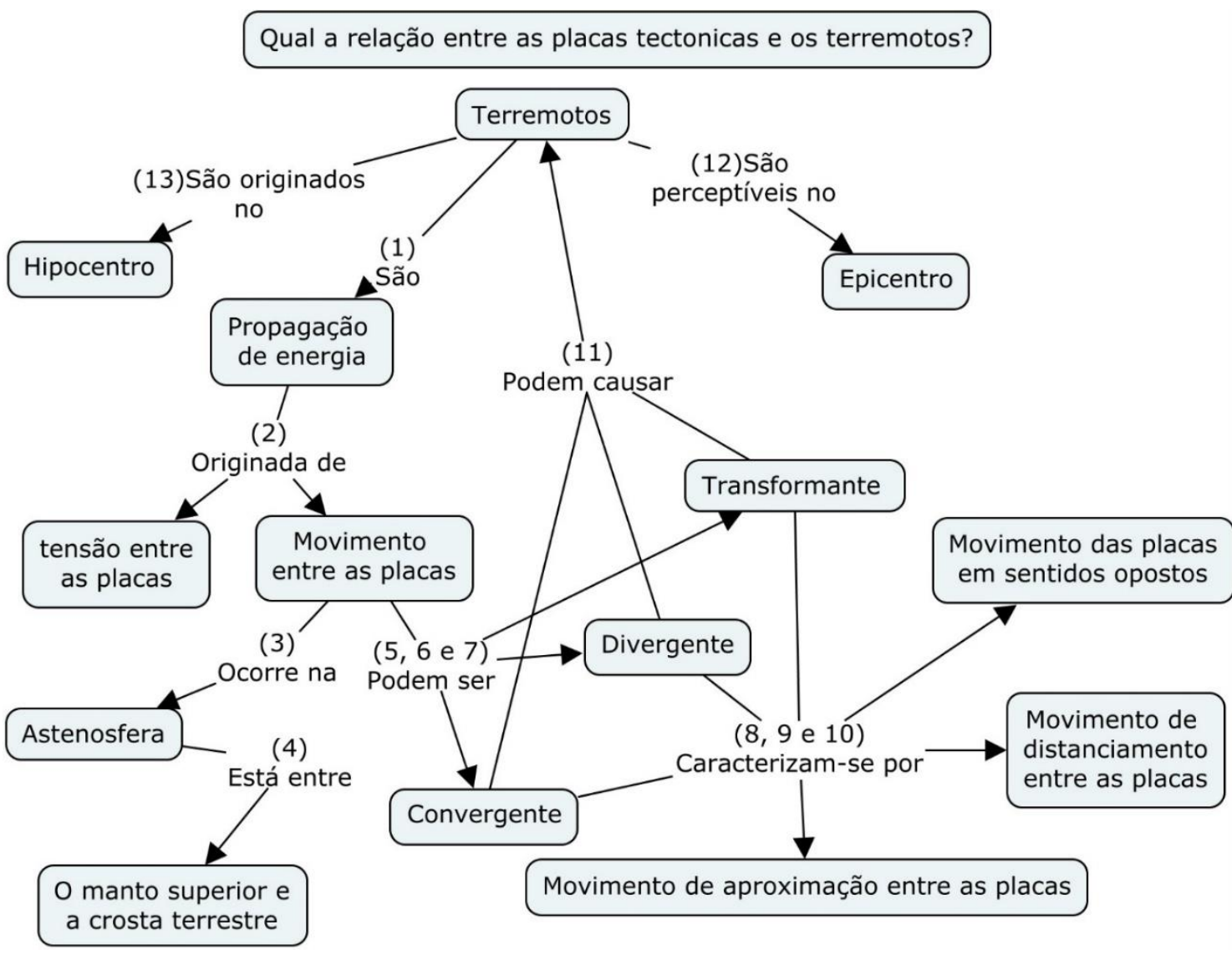

Fonte: arquivo do pesquisador (2016).

O mapa conceitual apresentado na Figura 3 passou pela revisão por pares, situação em que os próprios alunos deram sugestões para melhorar o mapa conceitual; cada par recebeu comentários de outros dois pares. 
Com a avaliação por pares, o aluno pôde analisar com mais detalhes a elaboração de seu mapa conceitual e receber sugestões dos alunos que têm domínio em tema semelhante ao seu. Observou-se que muitas sugestões foram coerentes e as duplas, em sua maioria, aceitaram as sugestões oferecidas pelos pares.

Uma análise mais detalhada do MC2 mostra um avanço tanto na estrutura quanto no conteúdo se comparado com o MC1. Os alunos numeraram os termos de ligação para facilitar a leitura do mapa conceitual, além de criar ligações cruzadas entre os conceitos, mostrando um bom domínio sobre o conteúdo estudado.

$\mathrm{Na}$ figura 4, observamos os alunos trabalhando no peer review no laboratório de informática. É importante destacar a dedicação e o compromisso dos alunos ao desenvolverem uma atividade de avaliação diferente do habitual.

\section{Figura 4: Análise e avaliação de um MC feito por uma dupla}

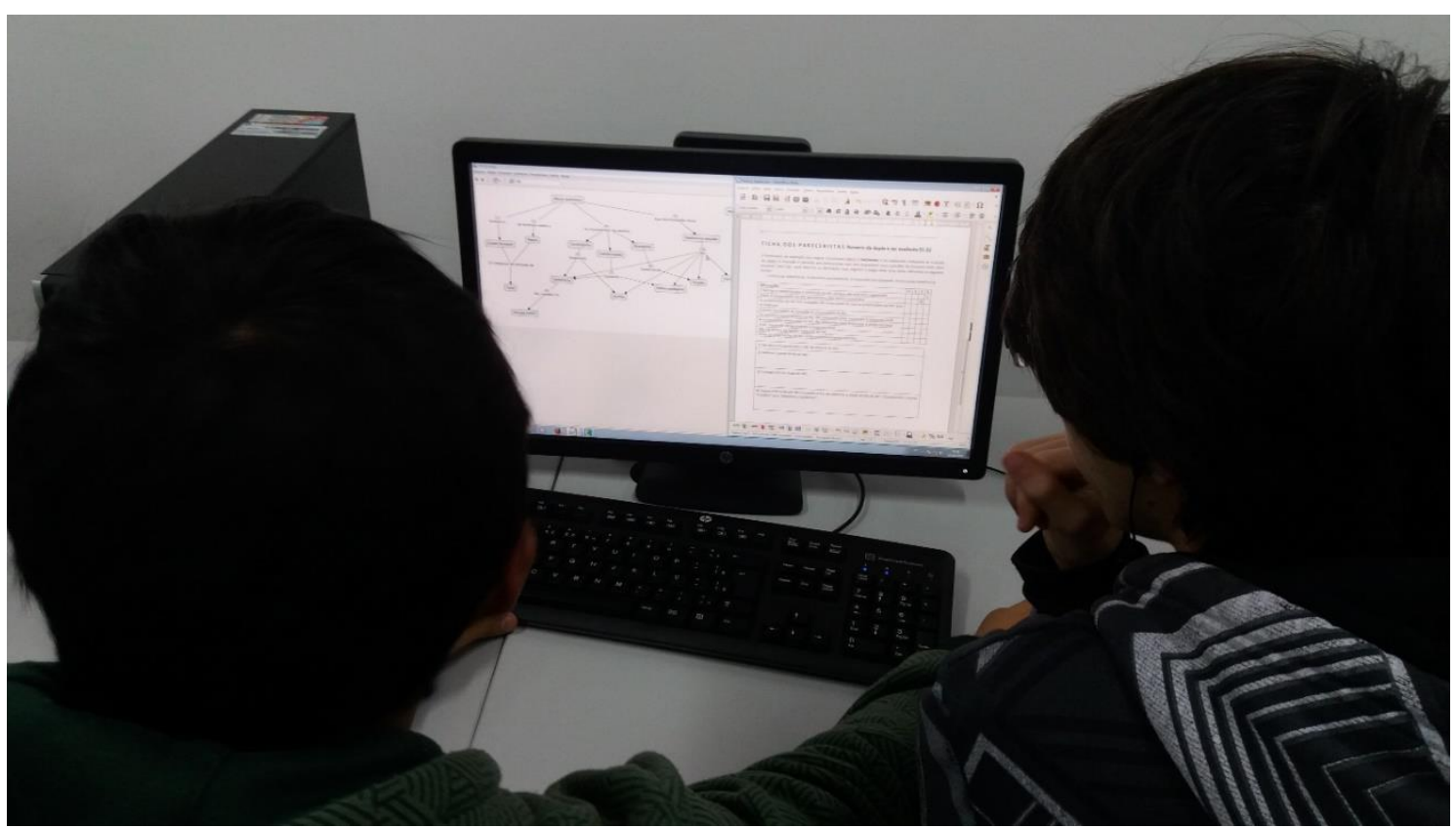

Fonte: arquivo do pesquisador (2016).

\section{Considerações Finais}

A técnica de mapeamento conceitual é uma ferramenta para organizar e representar conhecimento que pode levar a um alto nível de aprendizagem significativa. No entanto, a facilidade em entender como criar um mapa conceitual pode ser uma armadilha perigosa para professores, já que eles podem deduzir que seu uso na sala de aula é igualmente trivial. O sucesso no uso de mapas conceituais como uma estratégia 
inovadora na sala de aula depende de um período de treinamento na técnica, que deve envolver os professores (primeiro) e seus alunos (depois).

A estratégia de mapeamento conceitual descrita neste artigo pode ser usada na prática educacional de várias maneiras. Como uma tarefa introdutória, o mapeamento conceitual pode encorajá-los a verbalizar suas concepções, a debatê-las e a suscitar a necessidade de responder a perguntas e testar suposições. O mapa conceitual é um bom instrumento para os professores diagnosticarem rapidamente o uso de conceitos equivocados pelos alunos. A tarefa do mapa conceitual colaborativo ajudou os alunos a assumirem mais responsabilidade por sua própria aprendizagem durante o curso.

Além disso, a posição epistemológica tomada em classe pode influenciar a qualidade dos mapas conceituais e seu valor na aprendizagem: os mapas conceituais feitos em um contexto de sala de aula onde a maioria dos fatos são memorizados levam a resultados muito diferentes dos mapas conceituais feitos em uma sala de aula onde a aprendizagem significativa dos conceitos-chave é o objetivo central. A postura epistemológica, em conjunto com boas perguntas focais por parte do professor, pode levar a MC explicativos em oposição a MC descritivos.

Muitos alunos passaram a usar a estratégia de mapeamento conceitual em outras disciplinas. Por outro lado, devemos considerar essa experiência como ponto de partida, os alunos praticaram a estratégia por aproximadamente dois meses, é necessário mais tempo para criar alunos proficientes na estratégia de mapeamento conceitual.

\section{REFERÊNCIAS BIBLIOGRÁFICAS}

AUSUBEL, D. P. The acquisition and retention of knowledge: A cognitive view. Boston: Kluwer, 2000.

BLATCHFORD, P.; KUTNICK, P.; BAINES, E. e GALTO, M. Toward a social pedagogy of classroom group work. International Journal of Educational Research, no 39, 2003. p.153-172.

BOXTEL, C. V.; LINDEN J. V.; ROELOFS, E. e ERKENS, G. Collaborative concept mapping: Provoking and supporting meaningful discourse. Theory into Practice, 41, 2010. p. 40-46. 
BROWN, J. S.; COLLINS, A. e DUGUID, P. Situated cognition and the culture of learning. Educational Research, 18 (1), 1989. p. 32-42.

CAMPELO, L. F. e PICONEZ, S. C. B. Concept mapping in high school: an experience on teaching geography to measure deep, surface and non-learning outcomes. In: CAÑAS, A.; REISKA, P.; NOVAK, J. (Eds.) Innovating with Concept Mapping. Springer-Communications in Computer and Information Science (CCIS), 635, 2016. p.29-39.

CAÑAS, A. J.; NOVAK, J. D. e REISKA, P. Is my concept map large enough? In: CAÑAS, A.; REISKA, P.; NOVAK, J. (Eds.) Innovating with Concept Mapping. Springer - Communications in Computer and Information Science (CCIS), 635, 2016. p.128-143.

CAÑAS, A. J.; NOVAK, J. D. e REISKA, P. How good is my concept map? Am I a good Cmapper? Knowledge Management \& E-learning, 7(1), 2015. p. 6-19.

CASTROGiOVAnNi, A. C. et al. O Ensino de Geografia e suas Composições Curriculares. Porto Alegre: Mediação, 2014.

CASTELLAR, S. M. V. Geografia Escolar: contextualizando a sala de aula. Curitiba: CRV, 2014.

CASTELLAR, S. M. V. e VILHENA, J. Ensino de Geografia. São Paulo: Cengage Learning, 2010.

CAVAlCANTI, L. S. (Org.) Temas da Geografia na Escola Básica. Campinas: Papirus, 2013.

CORREIA, P. R. M.; CABRAL, G. e AGUIAR, J. G. Cmaps with Errors: why not? Comparing Two Cmap-based Assesment Tasks to Evaluate Conceptual Understanding. Springer, Communications in Computer and Information Science, no. 635, 2016. p. 115.

CORREIA, P. R. M.; CICUTO, C. e AGUIAR, J. G. Using Novakian Concept Maps to Foster Peer Collaboration in Higher Education. In: IFENTHALER, D. e HANEWALD, R. (Eds.). Digital Knowledge Maps in Education. 2014. p.195-217.

CORREIA, P. R. M. e MALACHIAS, M. E. I. Expanded Collaborative Learning and Concept Mapping: a road to empowering students in classrooms. In: TORRES, P. L. e MARRIOT, R. C. V. Handbook of research on collaborative learning using concept mapping. Hershey, PA: Information Science Reference, 2010. p. 283-300.

DILLENBOURG, P.; BAKER, M.; BLAYE, A. e O'MALLEY, C. The evolution of research on collaborative learning science. In: HEIMANN, P. \& SPADA, H. Learnings in human and machines: towards an interdisciplinary learning sciences. Oxford: Elsevier, 1995. p. 189-211 
DILLENBOURG, P. What do you mean by 'collaborative learning'? In:

DILLENBOURG, P. Collaborative Learning: cognitive and computational approaches, Oxford: Pergamon Press, 1999.

FISCHER, F.; BRUHN, J.; GRASEL, C. e MANDL, H. Fostering collaborative knowledge construction with visualization tools. Learning and Instruction, 12, 2002. p. 213-232.

HAMMES, C. C.; FORSTER, M. M. S.; CHAIGAR, V. A. M. Formação de Professores, Integração Curricular e a Geografia: o lugar escola como espaço de acontecimentos. In: CASTROGIOVANNI, A. C. et al. O Ensino de Geografia e suas Composições Curriculares. Porto Alegre: Mediação, 2014, p. 123-148.

HAY, D. B. Developing dialogical concept mapping as an e-learning technology. British Journal of Educational Technology, no39, 2008. p. 1057-1060.

KIMURA, S. Geografia no Ensino Básico - questões e propostas. São Paulo: Contexto, 2008.

KINCHIN, I. M.; DELEIJ, F. A. A. M. e HAY, D. B. The evolution of a collaborative concept mapping activity for undergraduate microbiology students. Journal of Further and Higher Education, 29, 2005. p. 1-14.

KINCHIN, I. M. Effective teacher $\leftrightarrow$ student dialogue: A model from biological education. Journal of Biological Education, 37, 2003, p. 110-113.

KINCHIN, I. M. e HAY, D. How a qualitative approach to concept map analysis can be used to aid learning by illustrating patterns of conceptual development. Educational Research, vol. 42, no. 1, Spring, 2000. p. 43-57.

MAIA, D. C. Ensino de Geografia em Debate. Salvador: EDUFBA, 2014.

MOON, B. M.; HANSBERGER, J. T. e TATE, A. Concept mapping in virtual collaboration environments. In: MOON, B. M; HOFFMAN, R. R.; NOVAK, J. D. e CAÑAS, A. J. (Eds.) Applied concept mapping: Capturing, analysing, and organizing knowledge, London, UK: CRC Press, 2011. p. 293-316.

MOREIRA, M. A. e MASINI, E. F. S. Aprendizagem Significativa: a teoria de David Ausubel. 2a ed. São Paulo: Centauro, 2006.

NOVAK, J. D. Learning, Creating and Using Knowledge: Concept Maps as Facilitative Tools in Schools and Corporations. 2a Taylor e Francis, New York, 2010.

NOVAK, J. D. e MUSONDA, D. A twelve-year longitudinal study of science concept learning. American Educational Research Journal, v.28, n.1, 1991. p. 117-153.

NOVAK, J. D. e CAÑAS, A. J. A Teoria Subjacente aos Mapas Conceituais e como Elaborá-los e Usá-los. Práxis Educativa, Ponta Grossa, v.5, n.1, jan-jun, 2010. p. 9-29. 
REGO, N.; CASTROGIOVANNI, A. C.; KAERCHER, N. A. (Orgs.) Geografia práticas pedagógicas para o ensino médio. Volume 2. Porto Alegre: Penso, 2011.

SCHWENDIMANN, B. A. Comparing Expert and Novice Concept Map Construction Though a Talkud Protocol. In: CAÑAS, A.; REISKA, P.; NOVAK, J. (Eds.)

Innovating with Concept Mapping. Springer - Communications in Computer and Information Science (CCIS), 635, 2016. p.16-28.

SOUZA SANTOS, B. Renovar a teoria crítica e reinventar a emancipação social. Tradução Mouzar Benedito. São Paulo: Boitempo, 2007.

TORRES, P. L. e MARRIOT, R. C. V. Handbook of research on collaborative learning using concept mapping. Hershey, PA: Information Science Reference, 2010.

VENTURI, L. A. B. Geografia: práticas de campo, laboratório e sala de aula. São Paulo: Sarandi, 2011.

Recebido em 06/07/2017

Aceito em: 18/10/2017 